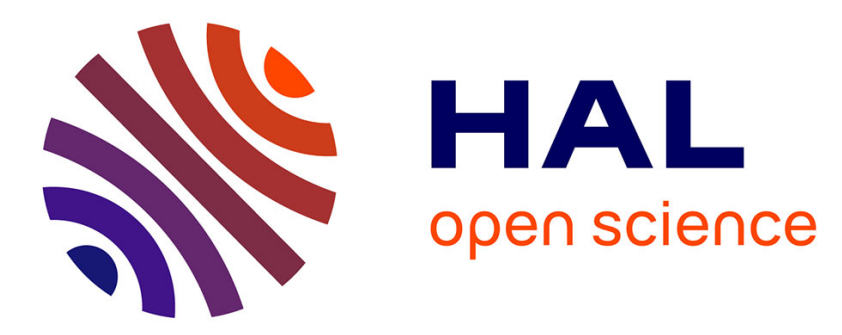

\title{
Magnetoresistance in an amorphous exchange-coupled bilayer
}

\author{
Thomas Hauet, F Montaigne, M. Hehn, Y Henry, S Mangin
}

\section{To cite this version:}

Thomas Hauet, F Montaigne, M. Hehn, Y Henry, S Mangin. Magnetoresistance in an amorphous exchange-coupled bilayer. Physical Review B: Condensed Matter and Materials Physics (1998-2015), 2009, 10.1103/PhysRevB.79.224435 . hal-01345332

\section{HAL Id: hal-01345332 \\ https://hal.science/hal-01345332}

Submitted on 13 Jul 2016

HAL is a multi-disciplinary open access archive for the deposit and dissemination of scientific research documents, whether they are published or not. The documents may come from teaching and research institutions in France or abroad, or from public or private research centers.
L'archive ouverte pluridisciplinaire HAL, est destinée au dépôt et à la diffusion de documents scientifiques de niveau recherche, publiés ou non, émanant des établissements d'enseignement et de recherche français ou étrangers, des laboratoires publics ou privés. 


\title{
Magnetoresistance in an amorphous exchange-coupled bilayer
}

\author{
T. Hauet, ${ }^{1,2}$ F. Montaigne, ${ }^{1}$ M. Hehn, ${ }^{1}$ Y. Henry, ${ }^{3}$ and S. Mangin ${ }^{1}$ \\ ${ }^{1}$ Institut Jean Lamour, CNRS, Nancy University, BP 70239, F-54506 Vandouvre les Nancy, France \\ ${ }^{2}$ San Jose Research Center, Hitachi Global Storage Technologies, San Jose, California 95135, USA \\ ${ }^{3}$ IPCMS-GEMM, 23 rue du Loess, BP 43, 67034 Strasbourg Cedex 2, France \\ (Received 7 March 2008; revised manuscript received 24 March 2009; published 29 June 2009)
}

\begin{abstract}
The effect of a magnetic domain wall on the electronic transport in disordered materials is studied in an exchange-coupled amorphous $\mathrm{Gd}_{40} \mathrm{Fe}_{60} / \mathrm{Gd}_{10} \mathrm{Fe}_{90}$ bilayer. In this amorphous system, the size and the shape of an interfacial domain wall is controlled by an external magnetic field. Current-in-plane transport measurements are performed on single GdFe layers, $\mathrm{Gd}_{40} \mathrm{Fe}_{60} / \mathrm{Gd}_{10} \mathrm{Fe}_{90}$ bilayer, and on a $\mathrm{Gd}_{40} \mathrm{Fe}_{60} / \mathrm{Si} / \mathrm{Gd}_{10} \mathrm{Fe}_{90}$ trilayer where the Si layer prevents the formation of the interfacial magnetic domain wall. Different contributions to the resistance are evidenced. In all types of samples, a linear positive magnetoresistance contribution is observed at high field which can be linked to the amorphous structure of the GdFe alloys. The comparison between the bilayer and the trilayer allows to eliminate this contribution and evidences that anisotropic magnetoresistance is the main effect induced by the interfacial domain wall. Beyond the anisotropic magnetoresistance signal, a supplementary negative magnetoresistance is evidenced. The origin of this effect is discussed qualitatively using previous theoretical predictions on magnetotransport through a magnetic domain wall in disordered metals.
\end{abstract}

DOI: $10.1103 /$ PhysRevB.79.224435

PACS number(s): 75.47.- m, 75.60.Ch, 73.23.-b

\section{INTRODUCTION}

Recent interest has grown concerning the interactions between a magnetic domain wall (DW) and a spin-polarized current due to fundamental discoveries opening fascinating perspectives for novel magnetic recording and device applications. ${ }^{1,2}$ For high current density, current-induced DW motion has been evidenced and linked to the torque provided by the spins on the magnetization when electrons flow between regions with different magnetization directions. ${ }^{3-5}$ For low spin-current density, the electronic spin flow is modified by the DW leading to additional resistance terms. Most of the previous experimental results concerning this last case have shown that a sufficiently thin DW generates an increase in resistivity. This effect originates from the incapacity of the spins to track the rotation of magnetic moments inside the wall, leading to a mixing of the two spin channels by spinflip scattering. ${ }^{6,7}$ Such GMR-type magnetoresistance has been observed in many systems such as films, ${ }^{8,9}$ wires, ${ }^{10,11}$ constrictions, ${ }^{12}$ or exchange-coupled multilayers. ${ }^{13}$ However, all of these studies involved crystalline or polycrystalline materials and, so far, no specific experimental study of the domain-wall influence on the magnetotransport in disordered systems has been done, although their transport properties are very different from the usual behaviors in crystalline materials.

In spite of many studies during the past half century, a complete understanding of transport mechanisms in disordered systems such as amorphous materials is still lacking because of the complexity and the wide spectrum of different tested materials. ${ }^{14}$ Strong structural disorder results in two major effects on electronic-transport properties. ${ }^{15,16}$ First, electron-electron interactions are enhanced by the diffusive nature of the transport and the very short mean-free path (of the order of few Angstroms). Coherent quantum interferences between electrons lead to an increase in effective
Coulomb-interactions time and, hence, to a conductivity correction, i.e., an increase in the resistance. ${ }^{17,18}$ Second, the reduction in the electronic-waves extension leads to a weak localization or a metal-insulator transition when the disorder is high enough. ${ }^{19}$ Since both of these features are strongly affected by a magnetic field or changes in ferromagnetic order, specific magnetoresistive effects are expected for these materials. In particular, the influence of a magnetic domain wall on disordered system electrical resistance has been studied theoretically. ${ }^{20-23}$ Contrary to what has been observed so far in crystalline magnets, the introduction of a DW in disordered metals is believed to decrease the resistivity due to either electron-electron interference destruction ${ }^{20}$ or weak localization suppression. ${ }^{21-23}$

Exchange-coupled bilayers using rare-earth transitionmetal alloys, such as $\mathrm{GdFe} / \mathrm{TbFe},{ }^{24,25} \mathrm{GdCo} / \mathrm{Co},{ }^{26}$ $\mathrm{DyFe}_{2} / \mathrm{YFe}_{2},{ }^{13,27}$ or $\mathrm{Gd}_{x} \mathrm{Co}_{1-x} / \mathrm{Gd}_{y} \mathrm{Co}_{1-y},{ }^{28}$ have been extensively used to study interfacial domain-wall (iDW) behaviors. In such bilayers, it is easy to control the size and the shape of a unique domain wall by applying an external magnetic field. This control has previously been used to elucidate some aspects of exchange bias. ${ }^{25,29}$ Here, we use an amorphous exchange-coupled $\mathrm{Gd}_{40} \mathrm{Fe}_{60} / \mathrm{Gd}_{10} \mathrm{Fe}_{90}$ bilayer to study domain-wall-induced magnetotransport effects in disordered metal systems. Magnetotransport measurements have been performed on single GdFe layers, $\mathrm{Gd}_{40} \mathrm{Fe}_{60} / \mathrm{Gd}_{10} \mathrm{Fe}_{90}$ bilayer and $\mathrm{Gd}_{40} \mathrm{Fe}_{60} / \mathrm{Si} / \mathrm{Gd}_{10} \mathrm{Fe}_{90}$ trilayer, where the Si layer prevents any direct exchange coupling between the magnetic layers. The comparison between the different structures allows distinguishing different magnetoresistance phenomena. At high fields a positive linear magnetoresistance is always observed which originates from the sample amorphous structure. The rotation of the magnetization inside the interface domain wall is found to generate anisotropic magnetoresistance (AMR). After having geometrically suppressed the AMR component, a negative magnetoresistance remains that is coherent with the theoretical predictions concerning the 


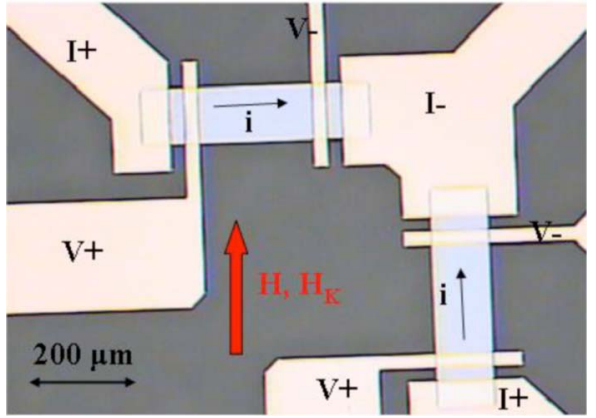

FIG. 1. (Color online) Optical-microscope image of two perpendicular $100 \times 400 \mu \mathrm{m}^{2}$ stripes designed in a $\mathrm{Gd}_{40} \mathrm{Fe}_{60} / \mathrm{Gd}_{10} \mathrm{Fe}_{90}$ bilayer to perform CIP four-probe measurement. The blue rectangles correspond to magnetic stripes. Pink regions are the gold pads. The red arrow indicates the orientation of the field $(H)$ and the anisotropy axis $\left(H_{K}\right)$.

effect of the domain wall on the diffusive transport of the electron inside amorphous GdFe alloys.

\section{EXPERIMENTAL DETAILS}

$\mathrm{Gd}_{40} \mathrm{Fe}_{60}(30 \mathrm{~nm})$ and $\mathrm{Gd}_{10} \mathrm{Fe}_{90}(30 \mathrm{~nm})$ ferrimagnetic amorphous layers were grown by coevaporation on substrates kept at $77 \mathrm{~K}$, under high vacuum (less than $10^{-8}$ mbar). For all temperatures up to $300 \mathrm{~K}$, the gadolinium contribution to the magnetization is dominant in $\mathrm{Gd}_{40} \mathrm{Fe}_{60}$ whereas the $\mathrm{Gd}_{10} \mathrm{Fe}_{90}$ magnetization is parallel to the iron spin sub lattice. ${ }^{30}$ For these two concentrations, no compensation point exists in the range from 300 to $5 \mathrm{~K}$ and the layers are purely amorphous, which is, for instance, no longer the case for Gd concentration less than $10 \%$ or higher than $90 \%$. Two samples, Glass $\| \mathrm{Gd}_{40} \mathrm{Fe}_{60}(30 \mathrm{~nm}) / \mathrm{Gd}_{10} \mathrm{Fe}_{90}$ $(30 \mathrm{~nm}) / \mathrm{Si} \quad(10 \mathrm{~nm})$ and Glass $\| \mathrm{Gd}_{40} \mathrm{Fe}_{60} \quad(30 \mathrm{~nm}) / \mathrm{Si}$ $(30 \mathrm{~nm}) / \mathrm{Gd}_{10} \mathrm{Fe}_{90}(30 \mathrm{~nm}) / \mathrm{Si}(10 \mathrm{~nm})$, hereafter referred to as the coupled and uncoupled sample, respectively, were grown simultaneously to avoid any difference in the composition and thickness of the ferrimagnetic layers (a metallic mask covered the coupled sample during the Si layer deposition). For the coupled sample, the interfacial exchange coupling between the layers is dominated by the Fe-Fe ferromagnetic exchange interaction so that the magnetizations of $\mathrm{Gd}_{40} \mathrm{Fe}_{60}$ and $\mathrm{Gd}_{10} \mathrm{Fe}_{90}$ are antiferromagnetically coupled.

Magnetization measurements were performed for all samples in a commercial superconducting quantum interference device magnetometer. The transport measurements were performed for each sample on two $100 \times 400 \mu \mathrm{m}^{2}$ stripes perpendicular to each other, shaped by UV lithography and Ar etching (Fig. 1). This geometry allows measuring simultaneously the resistance for a current flowing parallel and perpendicular to the applied field. The field is kept parallel to the $\mathrm{Gd}_{40} \mathrm{Fe}_{60}$ anisotropy axis (Fig. 1). Current-inplane (CIP) magnetoresitive measurements were performed on the stripes with a $1 \mathrm{~mA}$ current at different temperatures from 5 to $300 \mathrm{~K}$, using a conventional four-probe method in a Quantum Design Physical Property Measurement System (PPMS). In the following, the magnetoresistances are defined as $(\Delta R / R)(H)=[R(H)-R(0)] / R(0)$.

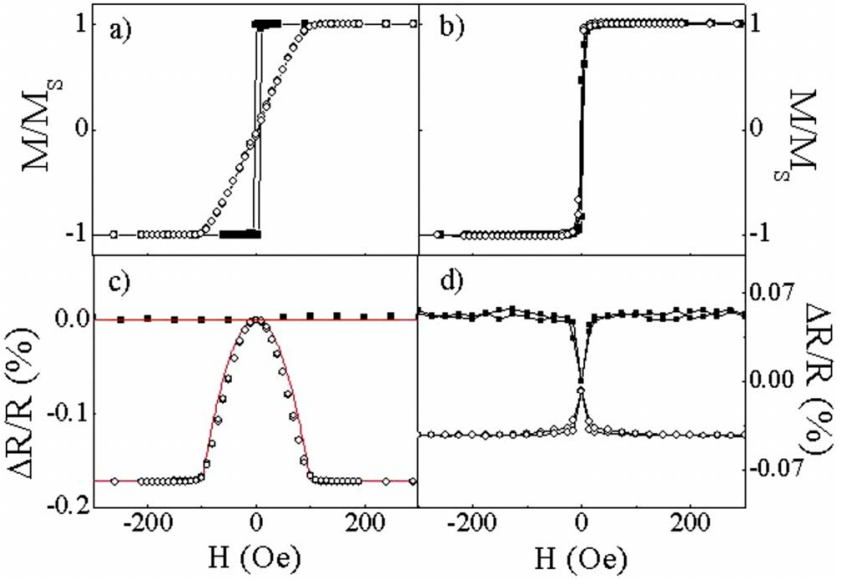

FIG. 2. (Color online) (a) $\mathrm{Gd}_{40} \mathrm{Fe}_{60}$ and (b) $\mathrm{Gd}_{10} \mathrm{Fe}_{90}$ normalized magnetization as a function of the field applied parallel (squares) and perpendicular (circles) to the anisotropy axis at $300 \mathrm{~K}$. (c) $\mathrm{Gd}_{40} \mathrm{Fe}_{60}$ and (d) $\mathrm{Gd}_{10} \mathrm{Fe}_{90}$ resistivity versus field for field applied parallel (squares) and perpendicular (circles) to the anisotropy axis kept parallel to the current at $300 \mathrm{~K}$. Red solid lines in (c) correspond to calculations explained in the text.

\section{RESULTS AND DISCUSSION}

\section{A. Single films}

Figures 2(a) and 2(c) present the normalized magnetization and magnetoresistance variations, measured at $300 \mathrm{~K}$, on a $\mathrm{Gd}_{40} \mathrm{Fe}_{60}$ alloy. This film shows a well-defined uniaxial anisotropy whose origin was previously related to the deposition geometry. ${ }^{31}$ We measured the magnetoresistivity in the case where the current flows along the uniaxial anisotropy. If a magnetic field is applied along this axis, a constant resistance $R_{\|}$is measured at low field $(H<1 \mathrm{kOe})$ whereas a continuous variation from $R_{\|}$to $R_{\perp}$ is observed as the external field is orientated perpendicular to the anisotropy axis (still in the film plane). The variations in resistivity in field are typical of the anisotropic magnetoresistance effect, ${ }^{32}$ which induces a dependence of the resistivity versus the angle $\theta$ between the magnetization and the current (here kept parallel to the anisotropy axis) such as $\rho_{\mathrm{AMR}}(\theta)=\rho_{\|} \cos ^{2} \theta$ $+\rho_{\perp} \sin ^{2} \theta$. The solid lines in Fig. 2(c) correspond to $\rho_{\mathrm{AMR}}(\theta)$ using the magnetization measurement presented in Fig. 1(a) and considering $\theta=\operatorname{arcos}\left[M(H) / M_{\mathrm{S}}\right]$, where $M_{\mathrm{S}}$ is the magnetization at saturation. The very good agreement between the $\rho_{\mathrm{AMR}}(\theta)$ and the experimental data indicates that AMR effect is sufficient to explain the $\mathrm{Gd}_{40} \mathrm{Fe}_{60}$ magnetoresistance variations for field $H<1 \mathrm{kOe}$. The same conclusion is obtained concerning $\mathrm{Gd}_{10} \mathrm{Fe}_{90}$ alloy whose magnetization and magnetoresistivity variations at low field are shown in Figs. 2(b) and 2(d). The hysteresis curves measured with field applied in the film plane along the easy and hard directions are almost identical. No strong uniaxial anisotropy exists for the $\mathrm{Gd}_{10} \mathrm{Fe}_{90}$ composition but the demagnetization field keeps the magnetic moments in the film plane. The resistivity measurements show two constant behaviors, with $\rho=\rho_{\|}$or $\rho=\rho_{\perp}$ when the field is applied parallel or perpendicular, respectively, to the current flow. Only small variations in resistivity due to AMR effect are observed close to zero field as the magnetization reverses. 


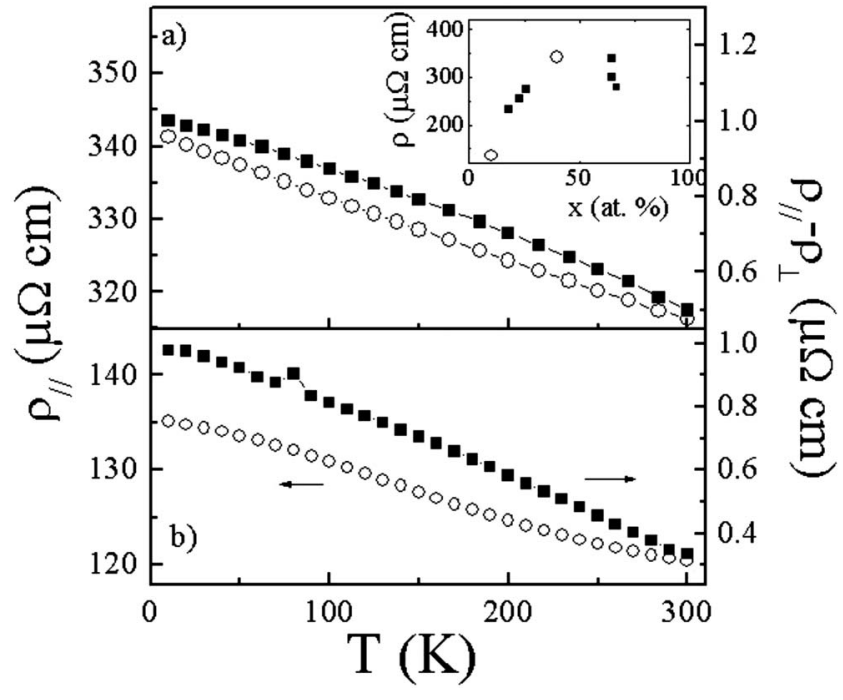

FIG. 3. (a) $\rho_{\|}$(circles) and $\rho_{\|}-\rho_{\perp}$ (squares) resistivity of $\mathrm{Gd}_{40} \mathrm{Fe}_{60}$ alloy as a function of temperature. Inset: $\rho_{\|}$versus concentration $x$ in $\mathrm{Gd}_{x} \mathrm{Fe}_{1-x}$ comparing values that we measured at $5 \mathrm{~K}$ (circles) and the ones shown in Refs. 33-37 (squares). (b) $\rho_{\|}$ (circles) and $\rho_{\|}-\rho_{\perp}$ (squares) resistivity of $\mathrm{Gd}_{10} \mathrm{Fe}_{90}$ alloy as a function of temperature.

In the inset of Fig. 3, we compare the resistivity $\rho_{\|}$for $\mathrm{Gd}_{40} \mathrm{Fe}_{60}$ and $\mathrm{Gd}_{10} \mathrm{Fe}_{90}$ that we measured with previous values reported in the literature on amorphous $\mathrm{Gd}_{x} \mathrm{Fe}_{1-x}$ films. ${ }^{33-37}$ These resistivities are high as compared to usual crystalline or polycrystalline ferromagnetic films but are common for amorphous or disordered materials. ${ }^{16,38}$ The longitudinal resistivity $\rho_{\|}$and the resistivity difference $\rho_{\|}-\rho_{\perp}$ evolutions for $\mathrm{Gd}_{40} \mathrm{Fe}_{60}$ and $\mathrm{Gd}_{10} \mathrm{Fe}_{90}$ single films are presented as a function of temperature in Figs. 3(a) and 3(b). A decrease in $\rho_{\|} \rho_{\perp}$ is observed for both alloys as the temperature increases. Such behavior is a common feature for both amorphous and crystalline metals. ${ }^{39}$ However, unlike in single-crystal and polycrystalline films, the longitudinal resisistivity is also found to decrease as the temperature increases. Our data are in perfect quantitative agreement with previous measurements performed on amorphous $\mathrm{Gd}_{x} \mathrm{Co}_{1-x}$ alloys and described in Ref. 40. This linear variation in temperature observed in most of the normal and magnetic amorphous metals, as well as the high value $\rho_{\|}>100 \mu \Omega \mathrm{cm}$, is usually explained by a Faber-Ziman-theory extension considering the influence of structural excitations, such as that of phonons on the multidiffusive transport. ${ }^{14,41}$

In Fig. 4 are presented the normalized resistance variations in $\mathrm{Gd}_{40} \mathrm{Fe}_{60}$ and $\mathrm{Gd}_{10} \mathrm{Fe}_{90}$ as a function of the magneticfield amplitude. Anisotropic magnetoresistance can account for the low-field behavior as described previously (Fig. 2). However AMR does not explain the positive and linear highfield slope shown in Fig. 4. From 1 to $70 \mathrm{kOe}$, an increase in resistivity is observed for both measurements with field parallel or perpendicular to the current flow. We note that $\Delta R / R$ at high field (e.g., $70 \mathrm{kOe}$ ) is ten times stronger in $\mathrm{Gd}_{10} \mathrm{Fe}_{90}$ than in $\mathrm{Gd}_{40} \mathrm{Fe}_{60}$. Moreover $\Delta R / R$ stays almost constant as a function of temperature for $\mathrm{Gd}_{40} \mathrm{Fe}_{60}$ whereas, in the case of $\mathrm{Gd}_{10} \mathrm{Fe}_{90}$, it decreases from $0.3 \%$ to $0.15 \%$ as the temperature decreases from 300 to $50 \mathrm{~K}$ and is constant for lower

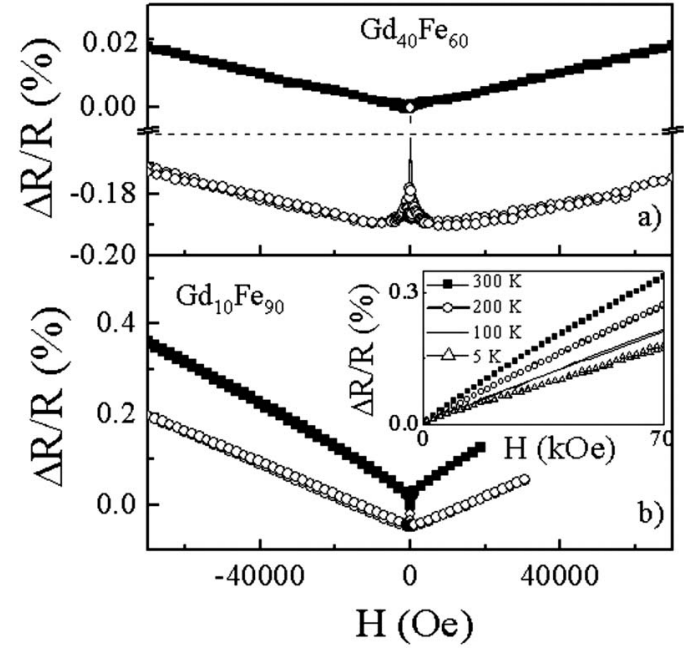

FIG. 4. Relative magnetoresistance variation measured on single (a) $\mathrm{Gd}_{40} \mathrm{Fe}_{60}$ layer and (b) $\mathrm{Gd}_{10} \mathrm{Fe}_{90}$ sample for field applied parallel (squares) and perpendicular (circles) to the anisotropy axis kept parallel to the current at $300 \mathrm{~K}$. Inset shows $\mathrm{Gd}_{10} \mathrm{Fe}_{90}$ relative magnetoresistance variation at different temperatures.

temperatures. Such an increase in the resistivity with field has already been studied in several magnetic-disordered materials such as $\mathrm{Fe}_{90-x} \mathrm{Co}_{x} \mathrm{Zr}_{10}{ }^{42} \mathrm{Fe}_{1-x} \mathrm{Co}_{x} \mathrm{Si}^{43} \mathrm{GdCo}_{3},{ }^{44}$ or more recently in $\mathrm{Fe}$ and $\mathrm{Ni}$ constrained thin films. ${ }^{45}$ To our knowledge, only electron-electron interference, i.e., Coulomb-effect-related theories, can account for such an unusual linear positive magnetoresistance for temperature up to $200 \mathrm{~K}$. This theory has particularly been successfully used in Ref. 44 to explain the high-field magnetoresistance behavior of amorphous $\mathrm{GdCo}_{3}$ films.

\section{B. Coupled and uncoupled samples}

\section{Magnetic properties}

Measurements of the magnetization as a function of the applied magnetic field performed on the $\mathrm{Gd}_{40} \mathrm{Fe}_{60}(30 \mathrm{~nm}) / \mathrm{Si}(30 \mathrm{~nm}) / \mathrm{Gd}_{10} \mathrm{Fe}_{90}(30 \mathrm{~nm})$ uncoupled sample at $50 \mathrm{~K}$ are presented in Fig. 5(a). Starting from saturation, two successive jumps correspond to the independent reversals of the $\mathrm{Gd}_{40} \mathrm{Fe}_{60}$ and $\mathrm{Gd}_{10} \mathrm{Fe}_{90}$. A minor loop describing the reversal of the softer $\mathrm{Gd}_{10} \mathrm{Fe}_{90}$ is shown as well. A full saturation is obtained at $250 \mathrm{Oe}$.

Figure 5(b) shows the magnetization of the coupled sample as a function of the field magnitude at $50 \mathrm{~K}$. A three step loop is observed. We used a one-dimensional (1D) micromagnetic simulation to characterize the magnetic configuration adopted by $\mathrm{Gd}_{40} \mathrm{Fe}_{60}(30 \mathrm{~nm}) / \mathrm{Gd}_{10} \mathrm{Fe}_{90}(30 \mathrm{~nm})$ as a function of the field. This model has already been successfully used in previous works and is described in detail in the Refs. 24 and 25. The depth-dependent magnetic profile is obtained by minimizing the surface energy including exchange coupling, anisotropy, and Zeeman energies. In the present work, the bilayer is considered to be made of 60 plans of laterally uniform magnetization. Temperature is only taken into account by changing the magnetization and the 


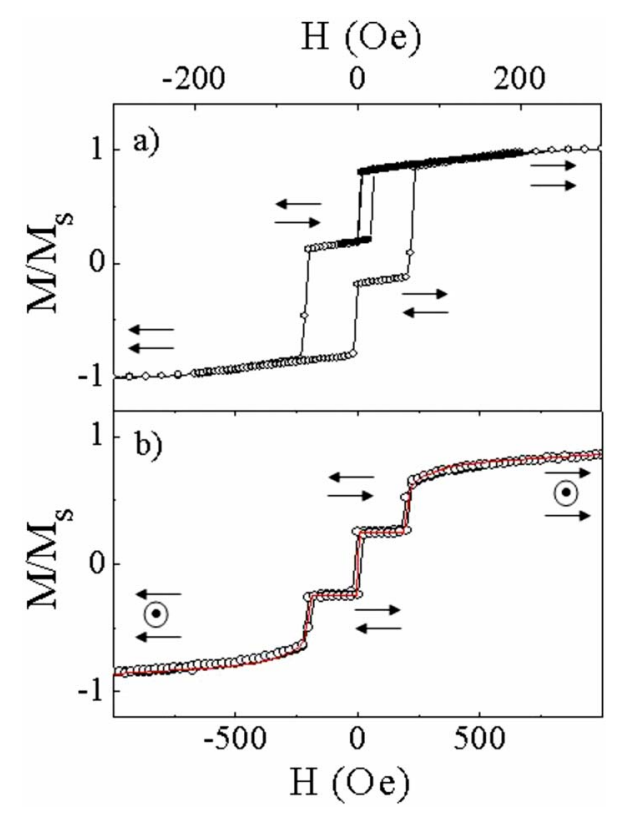

FIG. 5. (Color online) (a) Normalized magnetization measured at $50 \mathrm{~K}$ for $\mathrm{Gd}_{40} \mathrm{Fe}_{60} / \mathrm{Si} / \mathrm{Gd}_{10} \mathrm{Fe}_{90}$ as a function of the field. Square symbols correspond to a minor loop with $H_{\min }=30$ Oe. (b) Normalized magnetization measured at $50 \mathrm{~K}$ for $\mathrm{Gd}_{40} \mathrm{Fe}_{60} / \mathrm{Gd}_{10} \mathrm{Fe}_{90}$ as a function of the field. Solid line corresponds to the simulation.

anisotropy values. We used the following parameters to reproduce the magnetization curve presented in Fig. 5(b): the saturation magnetization $M_{\mathrm{Gd} 40 \mathrm{Fe} 60}=1050 \mathrm{emu} / \mathrm{cm}^{3}$ and $M_{\mathrm{Gd} 10 \mathrm{Fe} 90}=630 \mathrm{emu} / \mathrm{cm}^{3}$, the anisotropy constants $K_{\mathrm{Gd} 40 \mathrm{Fe} 60}=1.3 .10^{5} \mathrm{erg} / \mathrm{cm}^{3}$ and $K_{\mathrm{Gd} 10 \mathrm{Fe} 90}=3.1 .10^{4} \mathrm{erg} / \mathrm{cm}^{3}$, and the bulk-exchange stiffness $A_{\mathrm{Gd} 40 \mathrm{Fe} 60}=5.10^{-7} \mathrm{erg} / \mathrm{cm}$ and $A_{\mathrm{Gd} 10 \mathrm{Fe} 90}=2 \cdot 2 \cdot 10^{-7} \mathrm{erg} / \mathrm{cm}$. Magnetization and anisotropy values were measured on the single layers and are close to those used in our previous studies. ${ }^{24}$ The volumic exchange constant has been calculated using a mean-field theory developed in Ref. 30 and then adjusted by fitting experimental results. The interfacial antiferromagnetic exchange coupling across the $\mathrm{Gd}_{40} \mathrm{Fe}_{60} / \mathrm{Gd}_{10} \mathrm{Fe}_{90}$ interface is adjusted to $J=-1.5 \mathrm{erg} / \mathrm{cm}^{2}$. The calculated curve matches very well with the experimental data as shown in Fig. 5(b). Simulated magnetic configurations at $50 \mathrm{~K}$ are presented in the inset of Fig. 6. Since the magnetotransport effects mainly involve the transition-metal sublattice, ${ }^{46,47}$ we show only the Fe spins orientation as a function of the depth in the bilayer. At low field, the antiferromagnetic interface coupling energy dominates the total energy and the $\mathrm{Gd}_{40} \mathrm{Fe}_{60}$ and $\mathrm{Gd}_{10} \mathrm{Fe}_{90}$ stay antiparallel to each other. The $\mathrm{Gd}_{40} \mathrm{Fe}_{60}$ magnetization is kept parallel to the field direction to minimize the Zeeman term. Note that the absolute magnetizations at saturation and on the low-field plateaux are identical in the coupled and uncoupled sample which confirms identical magnetic layer thicknesses in both multilayers [Figs. 5(a) and 5(b)]. For fields larger than $250 \mathrm{Oe}$, an iDW is introduced in the depth of the bilayer resulting from the competition between the antiferromagnetically coupling and the Zeeman energy due to the field (inset of Fig. 6). The size of the domain wall decreases as the applied field increases from 250 to $70 \mathrm{kOe}$. In Fig. 6 are plotted the calculated domain-wall size as a

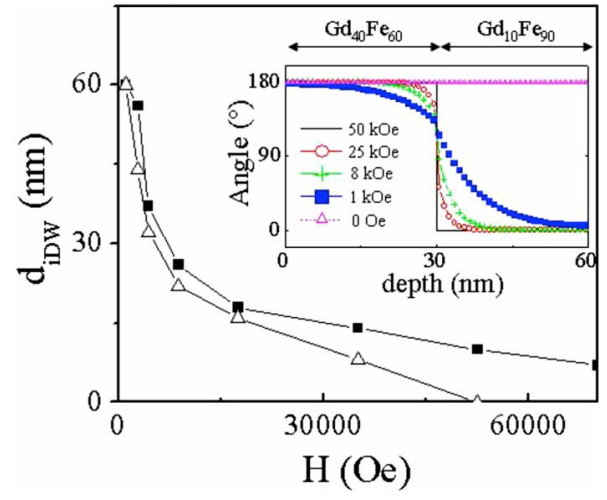

FIG. 6. (Color online) $\mathrm{Gd}_{40} \mathrm{Fe}_{60} / \mathrm{Gd}_{10} \mathrm{Fe}_{90}$ DW size $\left(d_{\mathrm{DW}}\right)$ as a function of the field simulated at 300 (square) and $50 \mathrm{~K}$ (triangle). Inset shows simulated $\mathrm{Fe}$ spins' orientation as a function of the depth in $\mathrm{Gd}_{40} \mathrm{Fe}_{60} / \mathrm{Gd}_{10} \mathrm{Fe}_{90}$ at $50 \mathrm{~K}$ for 0 (triangle), 1 (square), 8 (cross), 25 (circle), and $50 \mathrm{kOe}$ (line).

function of the field at 300 and $50 \mathrm{~K}$. This iDW size is extracted from the simulated magnetic configurations with the limits of the domain wall considered when the slope of the magnetic profile is less than 1 degree $/ \mathrm{nm}$. Simulations have been performed for a range from 300 to $5 \mathrm{~K}$. The DW compression as the field increases is similar for all temperatures but the domain-wall size for one particular field slightly decreases as the temperature decreases. As an intermediate conclusion, the bilayer system allows inducing and controlling the size of an interfacial domain wall as a function of the field amplitude. On the contrary, in the uncoupled sample, although the GdFe alloys are identical to those in the coupled sample, the absence of coupling prevents the formation of an iDW. Note that the absolute magnetizations at saturation and on the low-field plateaux are identical in the coupled and uncoupled sample which confirms identical magnetic-layer thicknesses in both multilayers [Figs. 5(a) and 5(b)].

\section{Magnetotranport properties}

Magnetoresistance measurements were performed on both uncoupled and coupled samples The absolute stripe resistance is about $70 \Omega$ (corresponding to an average resistivity of $210 \mu \Omega . \mathrm{cm}$ ). A difference in resistance of $1 \Omega$ was found between the coupled and uncoupled stripes. This variation can be explained by the Si layer resistance only present in the uncoupled sample and/or resistance contact discrepancies. The maximum difference measured between two perpendicular stripes of the same sample was found to be less than $0.1 \Omega$. We verified that these differences do not influence the following results. ${ }^{48}$

Figure 7(a) presents magnetoresistance variations for the uncoupled sample as a function of field at $50 \mathrm{~K}$. A linear increase in resistance is measured for both perpendicular stripes when the field increases up to $70 \mathrm{kOe}$. For fields $H$ $<1 \mathrm{kOe}$, a symmetric behavior in the two stripes' magnetoresistance of the uncoupled sample is presented in Fig. $7(\mathrm{c})$. This angular dependence is typical of the anisotropic magnetoresistance effect, ${ }^{32}$ which induces a dependence of the resistivity on the angle between the magnetization and the current. When the field is kept parallel to the ferrimag- 


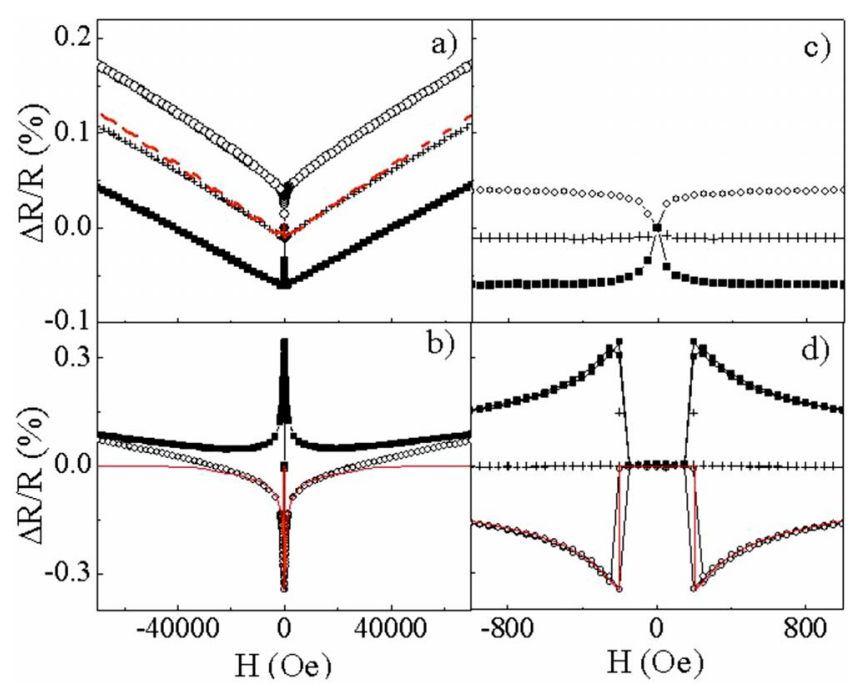

FIG. 7. (Color online) Relative magnetoresistance variation measured on (a) $\mathrm{Gd}_{40} \mathrm{Fe}_{60} / \mathrm{Si} / \mathrm{Gd}_{10} \mathrm{Fe}_{90}$ and (b) $\mathrm{Gd}_{40} \mathrm{Fe}_{60} / \mathrm{Gd}_{10} \mathrm{Fe}_{90}$ samples as a function of field applied parallel (circle) and perpendicular (square) to the current at $50 \mathrm{~K}$. Solid lines correspond to 1D simulations explained in the text. The dashed line in (a) represents the equivalent resistance obtained from single-layer measurements. (c) and (d) correspond to a zoom at low field of curves shown in (a) and (b), respectively. Cross symbol curves in (a) and (d) were calculated as the average of signals measured with field applied parallel (circle) and perpendicular (square) to the current.

netic layers' anisotropy axis, no AMR variation is expected. In the case where the current flows along the field direction, the resistivity is maximum $\left(\rho_{\|}\right)$and in the perpendicular case, the resistivity is minimum $\left(\rho_{\perp}\right)$. A small peak around zero fields can be observed, typical of AMR signal occurring during the magnetization reversal.

Magnetotransport measurements performed at $50 \mathrm{~K}$ on the coupled sample are shown in Figs. 7(b) and 7(d). To confirm that low-field magnetoresistance variations [Fig. 7(d)] are only due to AMR effect, the theoretical AMR values were calculated from the calculated magnetic configurations (Fig. 7). In our numerical simulation, the total conductance is considered as equivalent to that of parallel slides of the magnetic material at different depth $z$, having different magnetization orientation $\theta(z)$ with respect to the current direction. Its expression can then be written as follows: ${ }^{24,25}$

$$
\frac{1}{R}=\int_{z} \frac{d z}{\rho_{\|}+\left(\rho_{\perp}-\rho_{\|}\right) \sin ^{2} \theta},
$$

where the longitudinal resistivity $\rho_{\|}$and the resistivity difference $\rho_{\perp}-\rho_{\|}$values were measured on single layers. A good agreement is presented in Fig. 7(d) between the experimental data and the micromagnetic calculation in the case where the current flows along the magnetic-field direction. For 0 Oe $<|H|<250$ Oe, the two layer magnetizations are antiferromagnetically coupled and the AMR value is constant and maximum (The Fe magnetization is aligned with the current in the whole structure). At $|H|=250 \mathrm{Oe}$, since the DW is nucleated at the interface of the bilayer, a magnetization component appears perpendicular to the current direction and

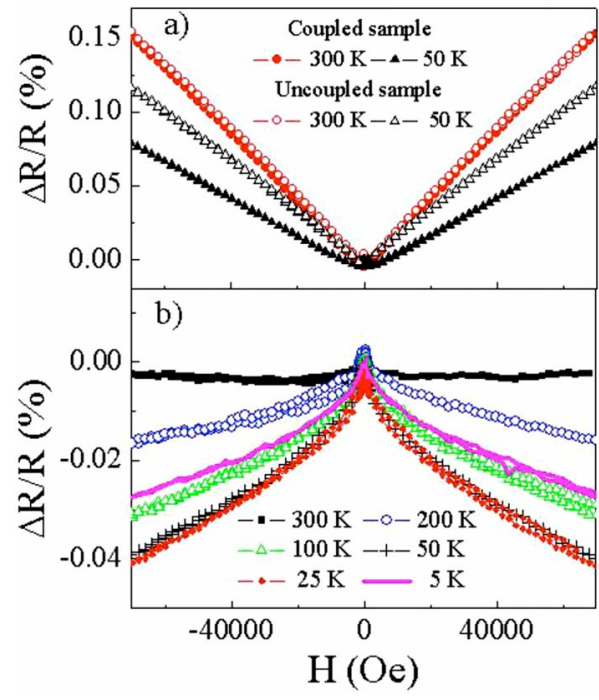

FIG. 8. (Color online) (a) Comparison between the average relative magnetoresistance of $\mathrm{Gd}_{40} \mathrm{Fe}_{60} / \mathrm{Gd}_{10} \mathrm{Fe}_{90}$ (full symbols) and $\mathrm{Gd}_{40} \mathrm{Fe}_{60} / \mathrm{Si} / \mathrm{Gd}_{10} \mathrm{Fe}_{90}$ (open symbols) measured at 300 (red circle) and $50 \mathrm{~K}$ (triangle). (b) Persistent relative magnetoresistance, i.e., difference between the average signal from the coupled sample and from the uncoupled sample, as a function of the field amplitude at 300 (black square), 200 (blue circle), 100 (green triangle), 50 (black cross), and $5 \mathrm{~K}$ (magenta solid line).

the AMR contribution jumps down. As the field increases, the DW extension shrinks; hence the AMR continuously increases and tends to reach its maximum value at high field, when the $\mathrm{Gd}_{40} \mathrm{Fe}_{60}$ and $\mathrm{Gd}_{10} \mathrm{Fe}_{90}$ magnetizations tend to become parallel. This AMR signal is typical of antiferromagnetically exchange-coupled bilayer, as already observed for $\mathrm{GdFe} / \mathrm{TbFe}^{25}$ or $\mathrm{GdFe} / \mathrm{FeSn}$ bilayers. ${ }^{49}$

The AMR signal amplitude measured at $50 \mathrm{~K}$ is about $0.3 \%$, i.e., larger than the high-field variations of $0.15 \% \mathrm{ob}-$ served on the uncoupled multilayer [Fig. 7(a)]. To further distinguish between the AMR and the other magnetoresistive contributions, we add for each sample the signal coming from both perpendicular stripes. Due to the AMR symmetry dependence, this procedure removes any AMR contribution as described in Refs. 7, 13, and 50. In Figs. 7(c) and 7(d) are presented the average value calculated as $(\Delta R / R)_{\text {average }}=\frac{1}{2}$ $\left[(\Delta R / R)_{\text {stripe } 1}+(\Delta R / R)_{\text {stripe } 2}\right]$ for the coupled and uncoupled sample, respectively (adding directly the resistances or the resistivities leads to the same results ${ }^{48}$ ). As expected, in both cases, the resulting average signal is constant over the whole range from -1 to $1 \mathrm{kOe}$ where the AMR contribution dominates. For higher field, a positive magnetoresistive signal persists either in the coupled or uncoupled samples [Fig. $8(\mathrm{a})$. This high-field linear positive magnetoresistance is similar to the one observed in single $\mathrm{Gd}_{40} \mathrm{Fe}_{60}$ and $\mathrm{Gd}_{10} \mathrm{Fe}_{90}$ (Fig. 3) and is related to the amorphous structure of the samples. In Fig. 7(a) are superimposed the data extracted from the uncoupled sample and the magnetoresistance variations calculated from the single $\mathrm{Gd}_{40} \mathrm{Fe}_{60}$ and $\mathrm{Gd}_{10} \mathrm{Fe}_{90}$ layers considered as in parallel electrical conduction. Similar slopes are observed for the uncoupled sample and singlelayer samples. Figure 8(a) presents the curves obtained after 
the AMR removal, at 300 and $50 \mathrm{~K}$, on coupled and uncoupled samples. Although the AMR exempted curves are almost identical at $300 \mathrm{~K}$, they show a clear difference at 50 $\mathrm{K}$, with a discrepancy of $0.04 \%$ at $70 \mathrm{kOe}$ between the coupled and uncoupled sample.

To better characterize this discrepancy as a function of temperature, we present in Fig. 8(b) the difference between the high-field persistent signal from the coupled sample and from the uncoupled sample versus field at different temperatures. A continuous nonlinear decrease in resistance is observed as the field increases up to $70 \mathrm{kOe}$. The amplitude of this effect is almost zero at $300 \mathrm{~K}$ and increases when the temperature decreases down to $25 \mathrm{~K}$ [Fig. 5(b)]. For lower temperatures, a surprising nonmonotonic behavior is observed and, e.g., the $5 \mathrm{~K}$ curve is closer to the $100 \mathrm{~K}$ signal [Fig. 8(b)].

\section{DISCUSSION}

The high resistivity values, as well as its temperature and its field dependence measured in single $\mathrm{Gd}_{40} \mathrm{Fe}_{60^{-}}$and $\mathrm{Gd}_{10} \mathrm{Fe}_{90}$-based films, are consistent with the amorphous structure of the film. However, some observations on the single films are still not explained. For instance, the variation in the high-field magnetoresistance with the Gd concentration in $\mathrm{G}_{x} \mathrm{Fe}_{1-x}$ alloys remain unclear; further systematic experimental and theoretical studies are needed in order to better understand the transport properties of amorphous $\mathrm{GdFe}$ alloys. Nevertheless, our measurements confirm previous results obtained on several disordered magnetic materials. To our knowledge, only theories involving electron-electron interactions, i.e., Coulomb effects, ${ }^{18}$ were found to fit with such behaviors, as already shown in $\mathrm{GdCo}_{3}$ alloy. ${ }^{44}$

The comparison between the $\mathrm{Gd}_{40} \mathrm{Fe}_{60} / \mathrm{Si}(30 \mathrm{~nm}) /$ $\mathrm{Gd}_{10} \mathrm{Fe}_{90}$ trilayers and the $\mathrm{Gd}_{40} \mathrm{Fe}_{60} / \mathrm{Gd}_{10} \mathrm{Fe}_{90}$ bilayers allows isolating the intrinsic influence of the domain wall on the magnetotransport. Indeed, an interface domain wall can develop in the bilayer because of the interface antiferromagnetic exchange coupling whereas the two layer magnetizations are kept uniform in the trilayer system. Moreover, since the magnetic materials are grown at the same time for both trilayer and the bilayer, a direct comparison between the two samples is justified. Moreover the size and shape of the domain wall may be well controlled by the external field.

After removing the "high-field" component of the magnetoresitance, identified as originating from the sample structural disordered, and the AMR signal, an additional magnetoresistance signal was highlighted. This signal corresponds to a nonlinear decrease in the resistance as the field increases from 0 to $70 \mathrm{kOe}$. For the most part, the amplitude of this resistance reduction increases as the temperature decreases.

Since no structural difference between coupled and uncoupled samples are expected, the additional negative nonlinear magnetoresistance should be explained by the presence of the iDW introduced in $\mathrm{Gd}_{40} \mathrm{Fe}_{60} / \mathrm{Gd}_{10} \mathrm{Fe}_{90}$. In that case, the decrease in the resistance with field comes from the decrease in the iDW thickness as the field increases. Considering previous theoretical results on domain-wall-induced magnetoresistance in disordered systems, such an evolution would reveal the influence of the exchange-field rotation inside the DW since the angle between two successive Fe spins increases as the external field increases (inset of Fig. 6). Indeed Lyanda-Geller et al. ${ }^{20}$ have calculated that the rotation of the internal exchange field inside the DW suppresses electron-electron interference in disorder ferromagnetic films and hence reduces the resistivity. Nevertheless, these authors ${ }^{20}$ have considered the case of electron-electron interaction effects in only $1 \mathrm{D}$ and two-dimensional models and no direct dependence as a function of temperature or domain-wall size is deductible.

\section{CONCLUSION}

In conclusion, the magnetoresistive properties of an interfacial domain-wall (iDW) nucleated in a $\mathrm{Gd}_{40} \mathrm{Fe}_{60} / \mathrm{Gd}_{10} \mathrm{Fe}_{90}$ bilayer have been studied and compared with those of $\mathrm{Gd}_{40} \mathrm{Fe}_{60} / \mathrm{Si}(30 \mathrm{~nm}) / \mathrm{Gd}_{10} \mathrm{Fe}_{90}$, where the Si layer prevents the formation of the iDW. At low field, the change in the interface domain-wall size gives rise to anisotropic magnetoresistance. For field higher than $1 \mathrm{kOe}$, all the samples present a positive magnetoresistance variation that appears to be an intrinsic feature of the GdFe amorphous single layers. After geometrical suppression of the AMR contribution, comparison between magnetoresistance of $\mathrm{Gd}_{40} \mathrm{Fe}_{60}$ / $\mathrm{Gd}_{10} \mathrm{Fe}_{90}$ and $\mathrm{Gd}_{40} \mathrm{Fe}_{60} / \mathrm{Si}(30 \mathrm{~nm}) / \mathrm{Gd}_{10} \mathrm{Fe}_{90}$ reveals a nonlinear decrease in the resistance in the coupled sample, whose magnitude increases with increasing the field and decreasing the temperature. This additional signal is consistent with the scenario of DW reducing electron-electron interaction, ${ }^{20}$ however, further theoretical investigations are required in order to confirm this assumption.

\section{ACKNOWLEDGMENTS}

We thank D. Ligiardi, S. Suire, D. Pierre, and F. Mouginet for help with experiments.
${ }^{1}$ D. A. Allwood, G. Xiong, C. C. Faulkner, D. Atkinson, D. Petit, and R. P. Cowburn, Science 309, 1688 (2005).

${ }^{2}$ S. S. P. Parkin, U.S. Patent No. 6834005 (16 December 2004).

${ }^{3}$ L. Berger, Phys. Rev. B 54, 9353 (1996).

${ }^{4}$ J. C. Slonczewski, J. Magn. Magn. Mater. 159, L1 (1996).

${ }^{5}$ M. Klaui, C. A. F. Vaz, J. A. C. Bland, W. Wernsdorfer, G. Faini,
E. Cambril, L. J. Heyderman, F. Nolting, and U. Rudiger, Phys. Rev. Lett. 94, 106601 (2005); M. Klaui, P. O. Jubert, R. Allenspach, A. Bischof, J. A. C. Bland, G. Faini, U. Rudiger, C. A. F. Vaz, L. Vila, and C. Vouille, ibid. 95, 026601 (2005).

${ }^{6}$ P. M. Levy and S. Zhang, Phys. Rev. Lett. 79, 5110 (1997).

${ }^{7}$ M. Viret, D. Vignoles, D. Cole, J. M. D. Coey, W. Allen, D. S. 
Daniel, and J. F. Gregg, Phys. Rev. B 53, 8464 (1996).

${ }^{8}$ J. F. Gregg, W. Allen, K. Ounadjela, M. Viret, M. Hehn, S. M. Thompson, and J. M. D. Coey, Phys. Rev. Lett. 77, 1580 (1996).

${ }^{9}$ C. H. Marrows and B. C. Dalton, Phys. Rev. Lett. 92, 097206 (2004).

${ }^{10}$ R. Danneau, P. Warin, J. P. Attané, I. Petej, C. Beigné, C. Fermon, O. Klein, A. Marty, F. Ott, Y. Samson, and M. Viret, Phys. Rev. Lett. 88, 157201 (2002)

${ }^{11}$ C. Hassel, M. Brands, F. Y. Lo, A. D. Wieck, and G. Dumpich, Phys. Rev. Lett. 97, 226805 (2006).

${ }^{12}$ S. Lepadatu and Y. B. Xu, Phys. Rev. Lett. 92, 127201 (2004).

${ }^{13}$ S. N. Gordeev, J.-M. L. Beaujour, G. J. Bowden, B. D. Rainford, P. A. J. de Groot, R. C. C. Ward, M. R. Wells, and A. G. M. Jansen, Phys. Rev. Lett. 87, 186808 (2001).

${ }^{14}$ P. J. Cote and P. J. Meisel, in Glassy Metals I, Topics in Applied Physics 46, edited by H. J. Guntherodt and H. Beck (Springer Verlag, Berlin, 1981), p. 141.

${ }^{15}$ N. F. Mott, Adv. Phys. 13, 325 (1964).

${ }^{16}$ N. F. Mott and E. A. Davis, Electronic Process Innoncrystalline Materials (Clarendon, Oxford, 1979).

${ }^{17}$ P. A. Lee and T. V. Ramakrishnan, Rev. Mod. Phys. 57, 287 (1985).

${ }^{18}$ B. L. Altshuler and A. G. Aronov, in Electron-Electron Interactions in Disordered Systems, edited by A. L. Efros and M. Pollak (North Holland, North-Holland, Amsterdam, 1985).

${ }^{19}$ B. J. Hickey, D. Greig, M. A. Howson, J. Phys. F: Met. Phys. 16, L13 (1986).

${ }^{20}$ Y. Lyanda-Geller, I. L. Aleiner, and P. M. Goldbart, Phys. Rev. Lett. 81, 3215 (1998).

${ }^{21}$ G. Tatara and H. Fukuyama, Phys. Rev. Lett. 78, 3773 (1997).

${ }^{22}$ P. A. E. Jonkers, S. J. Pickering, H. De Raedt, and G. Tatara, Phys. Rev. B 60, 15970 (1999).

${ }^{23}$ P. E. Falloon, R. A. Jalabert, D. Weinmann, and R. L. Stamps, Phys. Rev. B 74, 144425 (2006).

${ }^{24}$ F. Montaigne, S. Mangin, and Y. Henry, Phys. Rev. B 67, 144412 (2003).

${ }^{25}$ Y. Henry, S. Mangin, T. Hauet, and F. Montaigne, Phys. Rev. B 73, 134420 (2006).

${ }^{26}$ J. E. Wegrowe, A. Comment, Y. Jaccard, J.-Ph. Ansermet, N. M. Dempsey, and J.-P. Nozières, Phys. Rev. B 61, 12216 (2000).

${ }^{27}$ K. Dumesnil, M. Dutheil, C. Dufour, and P. Mangin, Phys. Rev. B 62, 1136 (2000).

${ }^{28}$ J. L. Prieto, B. B. van Aken, J. I. Martin, A. Perez-Junquera, G.
Burnell, N. D. Mathur, and M. G. Blamire, Phys. Rev. B 71, 214428 (2005).

${ }^{29}$ S. Mangin, F. Montaigne, and A. Schuhl, Phys. Rev. B 68, 140404(R) (2003).

${ }^{30}$ P. Hansen, C. Clausen, G. Munch, M. Rosenkranz, and K. Witter, J. Appl. Phys. 66, 756 (1989).

${ }^{31}$ S. Mangin, G. Marchal, W. Wernsdorfer, A. Sulpice, D. Mailly, and B. Barbara, J. Magn. Magn. Mater. 165, 13 (1997).

${ }^{32}$ A. P. Malozemoff, Phys. Rev. B 32, 6080 (1985).

${ }^{33}$ M. Hartmann and T. R. McGuire, Phys. Rev. Lett. 51, 1194 (1983).

${ }^{34}$ T. R. McGuire, R. J. Gambino, and R. C. Taylor, IEEE Trans. Magn. 13, 1598 (1977).

${ }^{35}$ G. Hadjipanaysis, S. C. Cornelison, J. A. Gerber, and D. J. Sellmyer, J. Magn. Magn. Mater. 21, 101 (1980).

${ }^{36}$ J. Kästner, H.-J. Shink, and E. F. Wassermann, Solid State Commun. 33, 527 (1980).

${ }^{37}$ T. R. McGuire, R. J. Gambino, and R. C. O'Handley, The Hall Effect and Its Applications, edited by C. L. Chien and C. R. Westgate (Plenum, New York, 1980).

${ }^{38}$ J. H. Mooij, Phys. Status Solidi A 17, 521 (1973).

${ }^{39}$ R. P. van Gorkom, J. Caro, T. M. Klapwijk, and S. Radelaar, Phys. Rev. B 63, 134432 (2001).

${ }^{40}$ H. Okuno and Y. Sakurai, J. Appl. Phys. 53, 8245 (1982).

${ }^{41}$ T. E. Faber and J. M. Ziman, Philos. Mag. 11, 153 (1965).

${ }^{42}$ P. D. Babu and S. N. Kaul, J. Non-Cryst. Solids 220, 147 (1997).

${ }^{43}$ N. Manyala, Y. Sidis, J. F. DiTusa, G. Aeppli, D. P. Young, and Z. Fisk, Nature (London) 404, 581 (2000).

${ }^{44}$ W. Maj, J. Magn. Magn. Mater. 82, 118 (1989); 89, 189 (1990).

${ }^{45}$ A. Gerber, I. Kishon, I. Ya. Korenblit, O. Riss, A. Segal, M Karpovski, and B. Raquet, Phys. Rev. Lett. 99, 027201 (2007).

${ }^{46}$ C. Bellouard, H. D. Rapp, B. George, S. Mangin, G. Marchal, and J. C. Ousset, Phys. Rev. B 53, 5082 (1996).

${ }^{47}$ X. Jiang, L. Gao, J. Z. Sun, and S. S. P. Parkin, Phys. Rev. Lett. 97, 217202 (2006).

48 "Décalage d'échange et magnétorésistances dans des bicouches à base de ferrimagnétiques amorphes couplés par échange" T. Hauet, Ph.D. thesis, Nancy University, 2006; http://tel.archivesouvertes.fr/tel-00105641/fr/

${ }^{49}$ F. Canet, S. Mangin, C. Bellouard, and M. Piecuch, Europhys. Lett. 52, 594 (2000).

${ }^{50}$ T. Hauet, F. Montaigne, M. Hehn, Y. Henry, and S. Mangin, Appl. Phys. Lett. 93, 222503 (2008). 